\title{
Dignity therapy for patients with brain tumours: qualitative reports from patients, caregivers and practitioners
}

\author{
Melissa B. Korman, Janet Ellis, Jennifer Moore, Denise Bilodeau, Sarah Dulmage, Margaret Fitch, \\ Christina Mueller, Arjun Sahgal, Claire Moroney \\ Sunnybrook Health Sciences Centre, Toronto, ON, Canada \\ Contributions: (I) Conception and design: C Moroney, J Ellis, M Fitch, J Moore, D Bilodeau, S Dulmage, C Mueller, A Sahgal; (II) Administrative \\ support: C Moroney, S Dulmage; (III) Provision of study materials or patients: D Bilodeau, A Sahgal; (IV) Collection and assembly of data: C \\ Moroney; (V) Data analysis and interpretation: MB Korman; (VI) Manuscript writing: All authors; (VII) Final approval of manuscript: All authors. \\ Correspondence to: Janet Ellis. Sunnybrook Health Sciences Centre, 2075 Bayview Ave., Room FG13, Toronto, ON, M4N 3M5, Canada. \\ Email: Janet.ellis@sunnybrook.ca.
}

Background Most individuals with brain tumours experience distress or cognitive impairment during the illness trajectory, potentially causing decreased quality of life, strain on interpersonal relationships and altered sense of self or of the world. Symptoms of brain tumour and treatment can cause increased reliance on others and decreased in sense of dignity. Dignity is an important consideration when caring for patients, as it can influence decisions at end-of-life. Dignity therapy (DT) is a therapeutic intervention that was developed for patients near the end of life. DT encourages the patient to reflect on the life lived, including important roles and sources of pride, resulting in the development of a 'Legacy Document'. DT has been shown to enhance quality of life and dignity, and reduce psychological and existential distress for patients at the end-of-life. There is little literature on the effectiveness of DT, or other quality of life interventions, in brain tumour populations; This paper reports on the feasibility of conducting DT with this population, and presents qualitative data gathered from patients with brain tumours who participated in DT, their caregivers, and their Dignity Therapists.

Methods: Participants were recruited from the Odette Cancer Centre in Toronto. One of five Dignity Therapists conducted the intervention; time data was logged. Immediately after the intervention, patient participants, their caregivers, and Dignity Therapists were sent an open-ended, self-report survey about their experience with DT. Qualitative content analysis was conducted by an impartial reviewer. Average time taken to conduct the intervention was determined.

Results: Fifteen out of the 17 recruited participants (88\%) completed the intervention; 2 were unable to complete the intervention due to progressing disease. Qualitative data was categorized according to two main areas of interest: Acceptability and Impact. Four participants, 5 caregivers and 4 care providers completed the qualitative surveys. All 4 patient participants reported benefits of DT that related to communication and/ or advanced care planning (ACP). Dignity therapists felt that the impact on their patients was positive, and reported satisfaction as a clinician.

Conclusions: The low attrition rate for the intervention suggests that DT is feasible in this population, though the required time to complete DT might be difficult for healthcare practitioners to provide within the recommended timeframe for this therapy. Positive qualitative reports on the effect of DT from patients, caregivers and dignity therapists alike indicate that DT is a promising intervention for this demographic.

Keywords: Quality of life; psychotherapy; brain neoplasms; psycho-oncology; self concept; respect

Submitted Mar 05, 2020. Accepted for publication Aug 28, 2020.

doi: 10.21037/apm-20-553

View this article at: http://dx.doi.org/10.21037/apm-20-553 


\section{Introduction}

Approximately 3,000 Canadians are diagnosed annually with primary brain tumours, and $20-40 \%$ of people with all types of cancer develop secondary brain tumours (1). Brain tumours cause a disproportionate share of cancer morbidity and mortality, potentially instilling fear in patients upon diagnosis, and a need for effective advanced care planning (ACP) conversations (2). Brain tumours are associated with loss of autonomy, social difficulty, pain, and existential or spiritual challenges, which can result in decreased quality of life and sense of dignity (2-6). Research has shown that individuals with brain tumours experience greater distress, or experience distress earlier in the illness trajectory than those with other cancers $(4,6)$. Up to $74 \%$ of people with brain tumours will experience some distress (existential, emotional, or physical) or cognitive impairment (of memory or concentration) throughout the illness trajectory (7). Existential distress is a process of deep spiritual questioning about life and death, which may challenge life-long beliefs (8). Emotional distress includes sadness, fear, and worry. Physical distress depends on the extent of damage to brain tissue according to tumour location and size, and can include seizures, motor deficits, loss of vision or hearing, and decline in communication and mobility $(9,10)$. Treatment for brain tumours may also result in distressing side-effects, including fatigue and impaired cognitive functioning $(3,9)$, possibly impacting independence, altering the view of self and causing increased feelings of distress or hopelessness (10). A brain tumour may cause change to personality, which might strain personal or professional relationships, causing social distress (11). Diagnosis and treatment of a brain tumour can thus alter the person's sense of the world and profoundly impact their quality of life.

When caring for the needs of people with incurable disease, the goal is to support dignity and meaning in life and death (10). As brain lesions have been associated with an altered sense of self (12-14), it is especially important for patients with brain tumors to be understood by those who care for them, not just as a patient but as a person. This can help to support a patients' sense of dignity and meaning. Loss of dignity has been shown to be a motivating factor of desire for hastened death, along with distress and demoralization. Dignity is a key component influencing distress and decisions at end-of-life (15).

End-of-life decision making and ACP are both influenced by a patients' level of illness understanding.
Unfortunately, many people with cancer are not fully aware of their prognosis, even at the end of life. Further, a literature review including a total of 4,686 participants with brain tumours revealed a low rate of use of ACP discussions or interventions at the end-of-life, and few reports on the effect of ACP on patient and caregiver confidence, wellbeing and quality of life (16). ACP is a critical element of quality cancer care, as it can help to improve patient autonomy and guide delivery of end-of-life care (17). Work to evaluate the use of such conversations in brain tumour populations has revealed low uptake, highlighting the need for system-wide change in practice (17). When people are able to prepare for the end-of-life, and plan and accept their dying, they can experience a better quality of death. ACP lowers hospital readmission rates and intensive care unit utilization, and according to caregivers allows their loved one to have a dignified death, with improved caregiver satisfaction with end-of-life care $(16,18)$. Illness understanding conversations and earlier preparation for death with ACP need to occur before cognitive decline, to honor a person's autonomy and choices. As well, interventions that support dignity and decrease distress are much needed in this population.

There is limited literature on improving psychosocial quality of life for patients with brain tumours, as most quality of life intervention studies mainly include participants with other cancers, with only a few with brain tumours (19). Research is needed to evaluate the impact of short-term interventions on quality of life. Although interventions such as cognitive behavioural therapy and existential psychotherapy are effective in treating depression and anxiety at end-of-life, these interventions can take months, and have high attrition rates in patients with brain tumours due to cognitive and physical deterioration near end-of-life (19-22). Brief interventions are more likely to be completed by patients, and to be offered by busy healthcare practitioners.

One such brief intervention, entitled dignity therapy (DT), was developed by Harvey Chochinov. There is little literature on the effectiveness of DT in patients with brain tumours. DT has the advantage of being a 2-session therapy, generally completed in 1-2 weeks, and has yielded positive results at end-of-life, such as enhanced quality of life and dignity, and reduced psychological and existential distress $(15,23)$. It encourages the patient to reflect on aspects of their life most significant to them, what they want remembered, past roles, sources 
of pride, accomplishment and meaning, as well as unfinished business and sense of legacy, resulting in the development of a 'Legacy Document', which can act as a memoir for the patient's family (23). This promotes a sense of continuity for the person at end-of-life (13). In one study evaluating DT, the majority of patients reported satisfaction with DT, and an increased sense of dignity and will to live (15); in another, after DT, sense of dignity was higher and desire for hastened death decreased (24). DT enables synthesis of one's life, and promotes a sense of meaning in the life lived (25). This paper reports on the feasibility of conducting DT with patients with brain tumours in the last year of life, as well as qualitative data on the acceptability and impact of DT collected from patients who participated in DT, their caregivers, and their Dignity Therapists. We present the following article in accordance with the SURGE reporting checklist (available at http://dx.doi.org/10.21037/apm-20553).

\section{Methods}

This study was approved by the Research Ethics Office at Sunnybrook Health Sciences Centre (FWA00000234). The study was conducted in accordance with the Declaration of Helsinki (as revised in 2013).

This pilot feasibility project aimed to determine whether DT was a feasible and acceptable intervention for patients with brain tumour.

\section{Participant recruitment}

Participants were recruited from the Central Nervous System (CNS) Oncology Clinic if they were: being treated for a recurrent and/or progressed primary malignant brain tumour, expected to live for at least two weeks and no more than one year, aware of his or her prognosis, interested in DT, willing to meet with the co-investigator at least three times over a period of six weeks*, English-speaking, and able to provide meaningful and reflective responses to the interview questions during the intervention (not limited by cognitive impairment based on clinical consensus of at least two of the co-investigators). Eligible patients were identified by one of the nurse practitioners or the social worker for the CNS site group during the patient's visit to the CNS clinic. The patient was invited to participate in the study by the social worker, nurse practitioner or the staff neuro-oncologist. While remaining sensitive to the fact that the patient may be feeling vulnerable at this time, DT was presented very briefly as one supportive care option available to the patient. If the patient or family expressed interest in DT, a follow-up phone call was made by the research nurse. Once the patient's interest in participating in the study was determined, he or she was asked to identify a family caregiver, who was also asked to participate. Consent forms were filled out at the next appointment or via email; Written informed consent was taken from all participants. $\left({ }^{*}\right.$ The intervention is carried out over a maximum of 6 weeks; timeline was sped up for participants with a shorter life expectancy).

\section{The intervention: DT}

The intervention was offered by one of five team members (a social worker, two nurse practitioners, one palliative care physician and one psychiatrist), all of whom trained in DT by the physician who developed the intervention (23). The dignity therapists met with each participant 1-2 times; to conduct the DT interview and to co-edit the legacy document. The interview was audio-recorded and transcribed. The interviewer edited the document according with the DT protocol, and then either met with the patient to co-edit or e-mailed the document to the patient and caregiver to review and edit. If e-mailing, the back and forth was conducted until the patient and caregiver were satisfied with the final document. The final document was either printed out or emailed to the participants.

\section{Data collection and analysis}

Both quantitative and qualitative data collection measures were used; this paper reports on answers to open-ended, qualitative self-report surveys completed within a few weeks post-intervention by patients, caregivers, and dignity therapists. These surveys inquired about the experience of providing/receiving DT (Table 1) and were mailed or e-mailed to patient and caregiver participants as well as the Dignity Therapists directly after finishing the intervention. No incentives were provided to participants for completing the survey. Qualitative content analysis was conducted by an impartial reviewer (not involved in conducting DT for this project).

The time and resources necessary to provide the intervention to each patient, up until the development of 
Table 1 Post-intervention open-ended survey questions

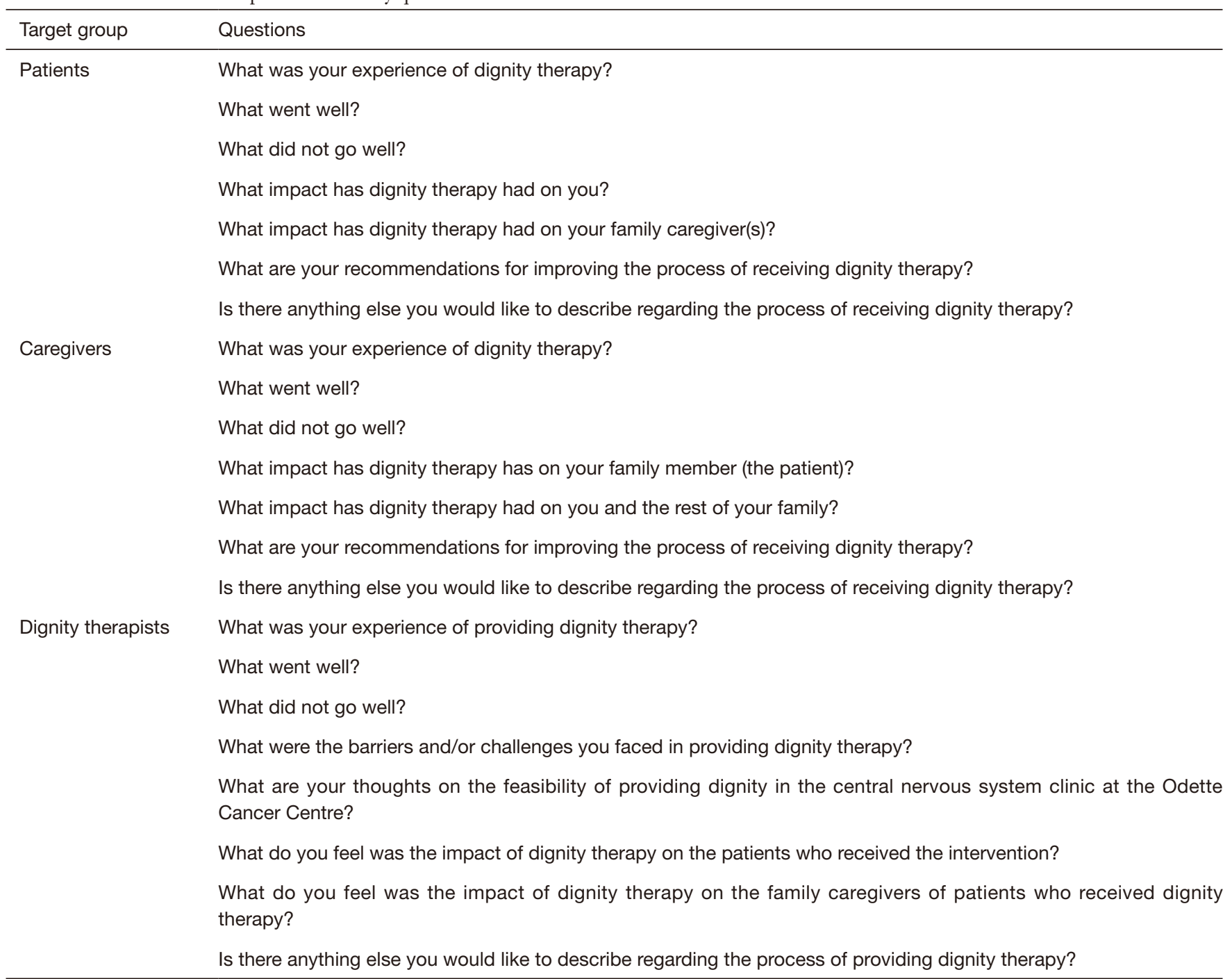

the legacy document, were tracked.

\section{Results}

\section{Quantitative feasibility data}

Fifteen out of the 17 recruited participants (88\%) completed the intervention; 2 were unable to complete the intervention due to progressing disease.

On average, it took $4.33-5.83$ hours of the dignity therapists' time, up until the co-editing process [see Table 2 for breakdown of time used], and 4-6 hours of paid transcriptionist time (costing $\$ 200$ per interview transcription).
Four participants, 5 caregivers and 4 care providers completed the qualitative survey portion. Qualitative data was categorized according to two main areas of interest: Acceptability and Impact.

\section{Acceptability}

All 4 patient participants reported benefits of DT; though 1 of the 4 patient participants criticized the amount of time taken for completion of the legacy document, and the quality of the final document, which was not the standard protocol of DT. Despite these criticisms, the same participant stated that DT "is an excellent idea and well worth continuing. In itself has been very helpful." Other participants reported that they "would recommend [DT] to those who are 
Table 2 Time taken to complete each part of the dignity therapy process, until co-editing

\begin{tabular}{ll}
\hline Activity & Time (minutes) \\
\hline Arranging interview & 10 \\
Preparing for interview & 10 \\
Conducting interview & $60-90$ \\
Initial editing & $180-240$ \\
\hline
\end{tabular}

willing...," that DT "is a great tool," and discussed being grateful for the opportunity to participate in DT. From the perspective of the dignity therapist, patients were "bighly motivated" to participate in DT and often "came prepared."

\section{Impact}

The dignity therapists felt that the impact on their patients was positive; they reported patients saying that the experience was "meaningful and helpful" and that it had a "positive impact." One of the dignity therapists, who also interacted with the caregivers, felt that "it has an even stronger impact on families as it leaves them with a small token of the patient once they are gone."

\section{Communication}

Patient and caregiver participants reported that DT was a useful tool in improving communication, allowing families to be more open about the patients' health status. Patients reported that DT, as well as the open conversations prompted by it, allowed acceptance of their situation: " $A s$ death is a part of my existence, it helps me to accept and prepare my children in the simplest form on what to expect." This open communication also helped interpersonal relationships. One caregiver stated that: "The interview was transformative. [The patient] spoke more during the course of the interview than be had for weeks leading up to it. On the following day be was much calmer and more connected with the kids than he had been for quite some time."

\section{$A C P$}

Communication was primarily improved through DT in allowing patients to speak more freely with family members about end-of-life. One patient stated being grateful for participating in the study because "even just doing the questionnaire, [...] it gave us a chance, because you don't necessarily want to talk about the end-of-life all the time and so it is a good prompt." Another patient explained that "DT has made us stop and think carefully about what we want, and we continue to be open and to discuss all our thoughts and concerns." For this patient, DT resulted in accessing palliative care services, having ACP conversations, and making end-of-life decisions earlier on than this patient would have otherwise.

\section{For the dignity therapist}

Overall, dignity therapists reported satisfaction as a clinician; "I felt like I was helping someone who was in distress in a very concrete way. Even though I wasn't able to take the problem away." Dignity therapists enjoyed the opportunity to help patients reflect on their lives and to get to know those patients better, feeling that it was both beneficial to themselves personally, as well as their work; "It enriches my work and reminds me of the importance of being curious about patients beyond their 'symptoms'." Beyond allowing them to provide improved care to their patients, they also reported feeling "surprised by how uplifting it was for me personally." They explained that in other cases, witnessing patients' distress caused feelings of demoralization and helplessness, whereas in DT interactions they witnessed distressed patients making meaning out of their lives, which was inspirational.

\section{Discussion}

DT is a brief therapeutic intervention that has been shown to enhance quality of life and dignity for patients near the end of life. This project gathered quantitative data on the feasibility of conducting DT, and qualitative data on the acceptability and impact of DT on patients with brain tumours. Based on the high rate of intervention completion (88\%) despite the late stage of illness, as well as positive feedback, DT appears to be an acceptable psychosocial intervention for this population. This is further solidified by dignity therapist reports of patients coming prepared (having reviewed the DT question protocol and having prepared answers) and being motivated to participate in the intervention. Consistent with the DT protocol, acceptability seems to hinge on rapid turn-around, with minimal time from recruitment to finalizing the legacy document. This is particularly important in this demographic, as patients with brain tumours can experience rapid cognitive decline towards end-of-life, so elongating the process might mean an inability to complete the intervention.

There is a gap in the research regarding the effectiveness 
and the mechanisms of change of current psychological interventions for patients with brain tumours. All those involved in providing DT to patients for this study reported a positive impact on their patients, based on both patient comments as well as their own observations. According to patient and caregiver reports, DT helped to improve communication, specifically aiding in their ability to have end-of-life and ACP conversations. The benefit of this was multifaceted; interpersonal relationships were positively affected, and patients were better able to accept their situation and sought specialized palliative care earlier on than they would have otherwise. Opening up these conversations might give the healthcare team and loved ones opportunities to provide emotional support to patients, making them feel less alone in their journey. This could also relieve the pressure of concerns that they may have otherwise been afraid to talk about. By addressing patients' distress and maintaining improved mental wellbeing, DT might enable patients to hold on to their sense of self further on into the illness trajectory. DT could potentially improve quality of life for patients through these mechanisms.

Though DT is a brief intervention for patients, it took 4.33-5.83 hours from the Dignity Therapists plus time for co-editing and transcriptionist hours. The entire DT intervention, including co-editing, is ideally completed within 1-2 weeks; this is a minimum of approximately 5 hours for each patient. DT was developed for patients near the end-of-life; considering the potential attrition due to cognitive deterioration in a brain tumour population, sessions would need to be booked as soon as possible once a patient consents to participating. Considering the high patient volumes seen in our regional cancer centre, it might not be feasible for busy clinicians to find enough time in their schedules to offer DT to patients, especially with late notice. That being said, longer term psychotherapy would often require more clinical hours allocated to each patient. If future larger-scale work demonstrates low attrition rate for the DT, and similar positive feedback in regards to both acceptability and impact, building capacity to offer DT on a larger scale could be a worthwhile endeavour.

In addition to the benefit to patients reported here, dignity therapists also reported experiencing personal satisfaction as a result of providing DT to their patients, suggesting that DT might be burnout-preventing for staff. Some were satisfied with an increased ability to provide quality care to their patients, for instance, one dignity therapist described feeling as though they were helping their patients in a concrete way, despite not having a cure to offer. Decreased self-efficacy is a risk factor for burnout, therefore, increased self-efficacy might be a protective factor. Similarly, one Dignity Therapist reflected on feeling demoralized or helpless when caring for patients with high distress and not being able to help them, whereas when providing DT to patients they felt inspired by their patients' ability to make meaning out of their lives, despite the circumstances. This further implies that conducting DT with patients might protect staff from burnout.

\section{Limitations and implications for future work}

The largest limitations were a small sample size and failure to collect data from a majority of participants that completed the intervention, which negatively impacts the generalizability of our findings. Future studies should systematically collect follow-up data sooner after completing the intervention, to avoid similar issues with patients' decline in cognitive status and inability to complete follow-up surveys. Future work should also use validated questionnaires to gather quantitative patient outcome data, in order to evaluate the impact of the intervention on quality of life, sense of dignity and meaning, and satisfaction with care. Based on the reported benefits to communication, specifically regarding end-oflife conversations, future work should also evaluate the effect of DT on patients and caregivers of those pursuing medical assistance in dying.

\section{Conclusions}

Positive reports on the effect of DT from patients, caregivers and dignity therapists alike identify DT as a promising intervention for patients with brain tumours. Specifically, the reportedly positive impact on communication and ACP might be mechanisms through which DT can improve psychosocial wellbeing and quality of life for patients with brain tumours.

\section{Acknowledgments}

We would like to thank the entire CNS site team for supporting and promoting this study.

Funding: This study was funded by the Central Nervous System Site Group at the Odette Cancer Centre. 


\section{Footnote}

Provenance and Peer Review: This article was commissioned by the Guest Editors (Jerome Graber, Hany Soliman) for the series "Palliative Care in Neuro-Oncology" published in Annals of Palliative Medicine. The article has undergone external peer review.

Reporting Checklist: The authors have completed the SURGE reporting checklist. Available at http://dx.doi.org/10.21037/ apm-20-553

Data Sharing Statement: Available at http://dx.doi. org/10.21037/apm-20-553

Peer Review File: Available at http://dx.doi.org/10.21037/ apm-20-553

Conflicts of Interest: All authors have completed the ICMJE uniform disclosure form (available at http://dx.doi. org/10.21037/apm-20-553). The series "Palliative Care in Neuro-Oncology" was commissioned by the editorial office without any funding or sponsorship. AS reports other from Abbvie, grants and other from Elekta/Elekta AB, other from Accuray Inc., other from Varian medical systems, other from BrainLAB, other from Merck, other from Roche, other from International Stereotactic Radiosurgery Society (ISRS), other from Medtronic Kyphon, other from VieCure, outside the submitted work. SD reports other from University of Toronto, George Brown College, Runnymede Healthcare Centre, and Centretown Community Health Centre, outside the submitted work. The authors have no other conflicts of interest to declare.

Ethical Statement: The authors are accountable for all aspects of the work in ensuring that questions related to the accuracy or integrity of any part of the work are appropriately investigated and resolved. The study was conducted in accordance with the Declaration of Helsinki (as revised in 2013). The study was approved by the Research Ethics Office at Sunnybrook Health Sciences Centre (FWA00000234) and informed consent was taken from all individual participants.

Open Access Statement: This is an Open Access article distributed in accordance with the Creative Commons Attribution-NonCommercial-NoDerivs 4.0 International License (CC BY-NC-ND 4.0), which permits the non- commercial replication and distribution of the article with the strict proviso that no changes or edits are made and the original work is properly cited (including links to both the formal publication through the relevant DOI and the license). See: https://creativecommons.org/licenses/by-nc-nd/4.0/.

\section{References}

1. Canadian Statistics Advisory Committee. Can J Stat 2018;46.

2. Rosenberg S. Dignity Therapy. Am J Psychiatry Residents Journal 2018;13:6-7.

3. Taphoorn MJB, Sizoo EM, Bottomley A. Review on Quality of Life Issues in Patients with Primary Brain Tumors. Oncologist 2010;15:618-26.

4. Edelstein K, Coate L, Massey C, et al. Illness intrusiveness and subjective well-being in patients with glioblastoma. J Neurooncol 2016;126:127-35.

5. Lovely MP. Quality of life of brain tumor patients. Semin Oncol Nurs 1998;14:73-80.

6. Ownsworth T, Nash K. Existential well-being and meaning making in the context of primary brain tumor: conceptualization and implications for intervention. Front Oncol 2015;5:96.

7. Randazzo D, Peters KB. Psychosocial distress and its effects on the health-related quality of life of primary brain tumor patients. CNS Oncol 2016;5:241-9.

8. Weisman AD, Worden JW. The Existential Plight in Cancer: Significance of the First 100 Days. Int J Psychiatry Med 1976-1977;7:1-15.

9. Chang S, Dunbar E, Dzul-Church V, et al. End-of-Life Care for Brain Tumor Patients. Neuro-Oncology GM Care 2015.

10. Mummudi N, Jalali R. Palliative care and quality of life in neuro-oncology. F1000Prime Rep 2014;6:71.

11. Liu R, Page M, Solheim K, et al. Quality of life in adults with brain tumors: Current knowledge and future directions. Neuro Oncol 2009;11:330-9.

12. Hiromitsu K, Asai T, Saito S, et al. Measuring the sense of self in brain-damaged patients: A STROBE-compliant article. Medicine (Baltimore) 2018;97:e12156.

13. Anderson-Shaw L, Baslet G, Villano JL. Brain neoplasm and the potential impact on self-identity. AJOB Neurosci 2010;1:3-7.

14. Sivertsen M, Normann B. Embodiment and self in reorientation to everyday life following severe traumatic brain injury. Physiother Theory Pract 2015;31:153-9.

15. Chochinov HM, Kristjanson LJ, Breitbart W, et al. 
Effect of Dignity Therapy on Distress and End-of-Life Experience in Terminally Ill Patients: A Randomised Controlled Trial. Focus. Lancet Oncol 2013;11:576-87.

16. Song K, Amatya B, Khan F. Advance care planning in patients with brain tumours: A prospective cohort study. J Cancer Res Ther 2015;3:85-91.

17. Song K, Amatya B, Voutier C, et al. Advance Care Planning in Patients with Primary Malignant Brain Tumors: A Systematic Review. Front Oncol 2016;6:223.

18. Sizoo EM, Taphoorn MJ, Uitdehaag B, et al. The endof-life phase of high-grade glioma patients: dying with dignity? Oncologist 2013;18:198-203.

19. Kangas M. Psychotherapy Interventions for Managing Anxiety and Depressive Symptoms in Adult Brain Tumor Patients: A Scoping Review. Front Oncol 2015;5:116.

20. Schuyler D, Brescia F. Psychotherapy of a Patient With Terminal Cancer. Prim Care Companion J Clin Psychiatry 2002;04:111-2.

Cite this article as: Korman MB, Ellis J, Moore J, Bilodeau D, Dulmage S, Fitch M, Mueller C, Sahgal A, Moroney C. Dignity therapy for patients with brain tumours: qualitative reports from patients, caregivers and practitioners. Ann Palliat Med 2021;10(1):838-845. doi: 10.21037/apm-20-553
21. Breitbart W, Gibson C, Poppito SR, et al. Psychotherapeutic Interventions at the End of Life: A Focus on Meaning and Spirituality. Focus 2007;5:451-8.

22. Applebaum AJ, Lichtenthal WG, Pessin HA, et al. Factors associated with attrition from a randomized controlled trial of meaning-centered group psychotherapy for patients with advanced cancer. Psychooncology 2012;21:1195-204.

23. Chochinov HM, Hack T, Hassard T, et al. Dignity Therapy: A Novel Psychotherapeutic Intervention for Patients Near the End of Life. J Clin Oncol 2005;23:5520-5.

24. Julião M, Oliveira F, Nunes B, et al. Effect of dignity therapy on end-of-life psychological distress in terminally ill Portuguese patients A randomized controlled trial. Palliat Support Care 2017;15:628-37.

25. Tatala $M$. The role of personal adjustment to developmental crises in improving quality of life. Int $\mathbf{J}$ Psychol Couns 2010;1:197-93. 